\title{
齿科氧化锆陶瓷水热稳定性研究进展
}

\author{
张晓旭 ${ }^{1,2}$, 朱东涁 ${ }^{1,2,3}$, 梁金生 ${ }^{1,2}$
}

(河北工业大学 1. 生态环境与信息特种功能材料教育部重点实验室; 2. 能源与环保材料研究所; 3. 机械工程学院, 天津 300130)

摘 要: 近年来, 齿科氧化锆陶瓷凭借高强韧性、良好生物相容性和美观自然色泽而成为牙齿临床修复的首选对象, 可用于修复、固定局部义齿和种植牙。然而, 在低温潮湿环境中氧化锆陶瓷易发生 t-m 相变老化, 服役寿命显著缩 短, 严重影响其临床稳定性。本文综述了氧化锆陶瓷低温老化的特点、机制及其老化动力学规律, 并介绍了表征 氧化锆低温老化现象的常规技术手段以及光学相干断层扫描、聚焦离子束等新方法; 总结了低温老化行为的主 要影响因素以及抗老化措施, 具体可通过调整材料体系、改进加工方式等来增强氧化锆的韧性, 解决其存在的低 温老化问题。随着齿科氧化锆陶瓷抗老化性能的提高以及健康功能化的未来需求, 其在齿科修复领域的应用将 会越来越广泛。

关 键 词: 齿科; 氧化锆; 低温老化; 老化动力学; 综述

中图分类号: TQ174 文献标识码: A

\section{Progress on Hydrothermal Stability of Dental Zirconia Ceramics}

\author{
ZHANG Xiaoxu $^{1,2}$, ZHU Dongbin $^{1,2,3}$, LIANG Jinsheng ${ }^{1,2}$ \\ (1. Key Laboratory of Special Functional Materials for Ecological Environment and Information of Ministry of Education, He- \\ bei University of Technology, Tianjin 300130, China; 2. Institute of Power Source and Ecomaterials Science, Hebei University \\ of Technology, Tianjin 300130, China; 3. School of Mechanical Engineering, Hebei University of Technology, Tianjin 300130, \\ China)
}

\begin{abstract}
In recent years, zirconia ceramic, as a preferential choice for teeth restorations, is used as fixed partial dentures and implants due to its excellent mechanical properties, favorable biocompatibility and aesthetic properties, thus significantly shortening the performance life and seriously damaging the reliabilities. However, zirconia ceramic easily occurs low temperature degradation (LTD) of $t-m$ transformation in humid environments. This paper illustrated the characteristics, mechanism and kinetics of LTD, as well as the conventional characterization methods of LTD phenomena and new methods such as optical coherence tomography and focused ion beam. It is also shown the main factors affecting the aging phenomenon, and emphasized the inhibition methods of LTD. By developing materials system and improving processing technology to enhance the strength, fracture toughness of zirconia and to solve the LTD of zirconia, and to meet the needs of people for their health functionalization, zirconia ceramic will be widely applied in the dental restoration field.
\end{abstract}

Key words: dentistry; zirconia; low temperature degradation; aging kinetics; review

收稿日期: 2019-08-09; 收到修改稿日期：2019-10-02

基金项目: 河北省自然科学基金(E2013202128,E2018202200) Natural Science Foundation of Hebei Province (E2013202128, E2018202200)

作者简介：张晓旭(1991-), 男, 博士研究生. E-mail: 15202203589@163.com ZHANG Xiaoxu (1991-), male, PhD candidate. E-mail: 15202203589@163.com

通讯作者：朱东涁，副教授. E-mail: zhudongbin@hebut.edu.cn ZHU Dongbin, associate professor. E-mail: zhudongbin@hebut.edu.cn 
在全球人口老龄化日益严重的趋势下，义齿需 求量增长迅猛。作为齿科修复材料, 首先需具有良 好的生物相容性, 无毒副作用; 其次还需足够的机 械强度 ${ }^{[1]}$, 承受口腔内咀嚼力的频繁变化; 同时, 伴随着人们对美观需求的提高, 要求齿科修复材料 的色泽与天然牙无限接近 ${ }^{[2-3]}$ 。

氧化锆陶瓷有着“陶瓷钢”的美誉 ${ }^{[4]}$, 具有优异 的力学性能(抗弯强度、断裂韧性)、良好的生物相 容性、色泽美观自然 ${ }^{[5-6]}$, 被视为理想的全瓷修复材 料, 逐渐代替氧化铝陶瓷, 在修复硬组织损伤方面 扮演着重要的角色 ${ }^{[7-8]}$ 。特别是氧化钎稳定氧化锆 $\left(\mathrm{Y}_{2} \mathrm{O}_{3}\right.$ stabilized tetragonal zirconia polycrystals, Y-TZP)陶瓷凭借 $\mathrm{t}-\mathrm{m}$ 相变增韧, 强度可达 $1000 \mathrm{MPa}$, 断裂韧性 $7.2 \mathrm{MPa} \cdot \mathrm{m}^{0.5}$ 。此外, 随着全瓷体加工技术 $\mathrm{CAD} / \mathrm{CAM}$ 的快速发展和成熟 ${ }^{[9-10]}$, 氧化锆陶瓷现 已被广泛用作齿科修复材料, 如牙根管、瓷桩、托 槽、牙冠及固定局部义齿(FDP)等 ${ }^{[11-12] 。 ~}$

然而, Kobayashi 等 ${ }^{[13]}$ 首次报道 Y-TZP 陶瓷在 $250{ }^{\circ} \mathrm{C}$ 潮湿环境下会发生缓慢的 $\mathrm{t}-\mathrm{m}$ 相变, 材料表 面出现微裂纹, 随后力学性能显著降低, 这种现象 通常称为低温老化 (Low temperature degradation, LTD)或水热老化(Hydrothermal aging, HA) ${ }^{[14-23]}$ 。现 在发现，低温(30 300 $\left.{ }^{\circ} \mathrm{C}\right)$ 潮湿条件下，如口腔或生 物体内环境, 氧化锆会自发地发生 $\mathrm{t}-\mathrm{m}$ 相变, 导致 力学性能和美学特性下降, 这种服役过程中的稳定 性问题，降低了临床应用效果。近年来，在保证其综 合力学性能的基础上, 学者针对这一问题以及如何 抑制低温老化现象进行了大量的体外时效研究。

掌握齿科氧化锆陶瓷老化机制及其老化动力学 规律, 对提高氧化锆陶瓷临床应用范围和耐久性具 有重要意义。因此, 本文首先介绍了氧化锆的低温 老化特点及机制，阐述了老化动力学规律; 其次, 概述了表征老化行为的技术手段; 最后, 分析和总 结了氧化锆老化的影响因素及预防老化行为的有效 途径, 并指出了齿科氧化锆陶瓷未来的研究方向。

\section{1 齿科氧化锆低温老化}

\section{1 低温老化的特点}

在低温潮湿环境下, 氧化锆发生 $\mathrm{t}-\mathrm{m}$ 相变老化 的本质是马氏体相变: 是非热力学、无扩散的晶体 结构变化。LTD 首先在材料表面发生 t-m 相变, 相 变伴随着体积澎胀使材料表面产生凸起及微裂纹、 美学性能退化; 随后水分子沿微裂纹渗透到基体内 部, 引起材料内部的氧化锆发生 $\mathrm{t}-\mathrm{m}$ 相变, 导致宏 观裂纹产生(图 1) ${ }^{[17]}$, 最终力学性能下降, 甚至引起 突发失效。
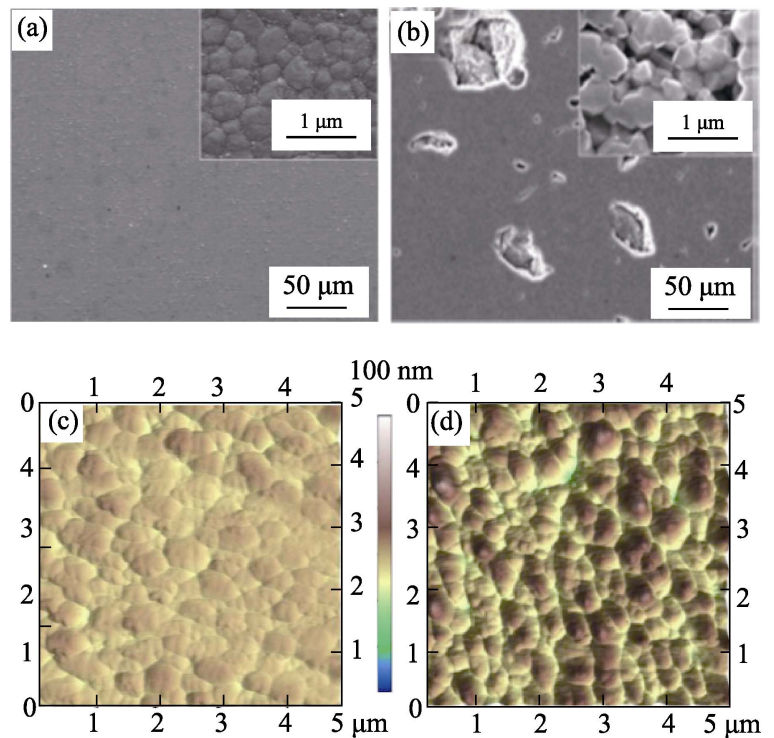

图 1 氧化锆老化前后的形貌对比 ${ }^{[17]}$

Fig. 1 SEM images of zirconia microstructure (a) without and (b) with LTD, and AFM images of zirconia (c) without and (d) with $\operatorname{LTD}^{[17]}$

经过大量的实验研究, LTD 过程的特征主要包 括 4 点: 1) LTD 是不导热的自催化过程, t-m 相变老 化是通过 $m$ 相的形核-长大(N-G)机制进行的 $\left.{ }^{[22]} ; 2\right)$ 老化 条件(温度、时间、水或水蒸气)改变会加速氧化锆 老化行为 $\left.{ }^{[21,23]} ; 3\right)$ 老化会导致材料 $m$ 相含量增加、 韧性下降、美学性能退化 ${ }^{[17]}$; 4)稳定剂含量和晶粒尺 寸直接影响氧化锆抗 LTD 性能 ${ }^{[18]}$ 。

\section{2 低温老化的机制}

氧化锆老化过程中 $\mathrm{H}_{2} \mathrm{O}$ 分子起诱导和加速作用 (图 2(a))。为解释 Y-TZP 老化中水分子的具体作用 机制, 研究者已经建立了多种老化模型 ${ }^{[18]}$ 。Lange 等 ${ }^{[19]}$ 认为 $\mathrm{H}_{2} \mathrm{O}$ 分子与四方晶粒中 $\mathrm{Y}_{2} \mathrm{O}_{3}$ 稳定剂反应 生成富含 $\mathrm{Y}(\mathrm{OH})_{3}$ 团簇, 耗尽四方相中 $\mathrm{Y}_{2} \mathrm{O}_{3}$ 稳定剂 导致亚稳态 $\mathrm{t}-\mathrm{ZrO}_{2}$ 自发地向 $\mathrm{m}-\mathrm{ZrO}_{2}$ 转变。Sato 等 ${ }^{[20]}$ 提出 $\mathrm{H}_{2} \mathrm{O}$ 分子与 $\mathrm{Zr}-\mathrm{O}-\mathrm{Zr}$ 键相互作用, 使其断裂并 形成 $\mathrm{Zr}-\mathrm{OH}$ 键, 导致四方相失稳发生相变。Yoshimura 等 ${ }^{[21]}$ 认为吸附的 $\mathrm{H}_{2} \mathrm{O}$ 分子在氧化锆表面解离并形 成 $\mathrm{Zr}-\mathrm{OH}$ 或 $\mathrm{Y}-\mathrm{OH}$ 键，导致表面晶格应力的产生和 应力积累, 触发了 $\mathrm{t}-\mathrm{m}$ 相变老化。然而这些机制均 未涉及晶粒尺寸及稳定剂对老化的影响。

目前，基于氧空位和水分子的点缺陷反应老化 机制比较完善并被广泛接受 ${ }^{[22-23]}$ (图 2(b))。该机制 认为老化过程可分为 5 个步骤: 1) $\mathrm{H}_{2} \mathrm{O}$ 分子化学吸 附在 $\mathrm{ZrO}_{2}$ 陶瓷表面 $\left.\left(\mathrm{H}_{2} \mathrm{O}_{\mathrm{ad}}\right) ; 2\right)$ 吸附的 $\mathrm{H}_{2} \mathrm{O}$ 分子和 $\mathrm{O}^{2-}$ 反应生成 $\mathrm{OH}_{\text {surf }}^{-}$3) $\mathrm{OH}^{-}$沿晶界扩散到 $\mathrm{ZrO}_{2}$ 材料 内部; 4) $\mathrm{OH}^{-}$占据氧空位 $\mathrm{V}_{\ddot{o}}$, 形成质子缺陷 $(\mathrm{OH})_{\mathrm{O}}$; 5) 当氧空位浓度减少至临界值时, $\mathrm{t}-\mathrm{ZrO}_{2}$ 失稳发生 $\mathrm{t}-\mathrm{m}$ 相变。该老化理论模型可用两个化学反应式描述: 


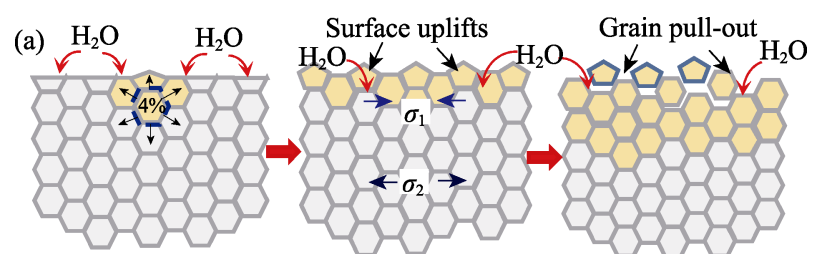

(1) $\square$ Tetragonal (2) $\square$ Monoclinic (3)

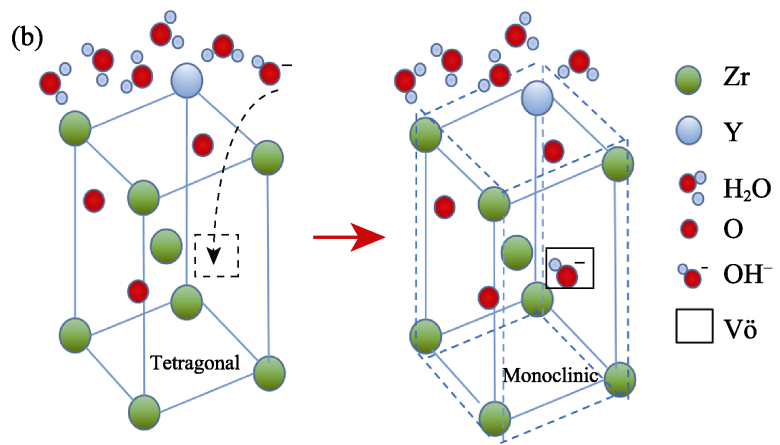

图 2 (a)氧化锆低温老化过程示意图及其(b)氧空位老化机 制 ${ }^{[23]}$

Fig. 2 (a) Schematic views of zirconia LTD process, and (b) oxygen vacancies of LTD mechanism ${ }^{[23]}$

$$
\begin{gathered}
\mathrm{H}_{2} \mathrm{O}_{\text {ad }}+\mathrm{O}_{\text {surf }}^{2-} \rightarrow 2 \mathrm{OH}_{\text {surf }}^{-} \\
2 \mathrm{OH}_{\text {surf }}^{-}+\mathrm{V}_{\ddot{\mathrm{o}}} \rightarrow(\mathrm{OH})_{\mathrm{O}}+\mathrm{S}_{\mathrm{O}_{\text {surf }}}^{\mathrm{X}}
\end{gathered}
$$

反应式(1)描述了 $\mathrm{ZrO}_{2}$ 空间结构键断裂的过程, 而 反应式(2)描述了羟基基团在 $\mathrm{ZrO}_{2}$ 表面扩散形成缺 陷的过程。在扩散过程中, $\mathrm{OH}^{-}$易被具有正电荷的晶 界吸引, 造成晶界处邻近的电荷层中氧空位的耗散, 最终触发 $\mathrm{t}-\mathrm{m}$ 相变。由于晶界处是氧空位主要聚集 存在的场所, 从而成为 $\mathrm{t}-\mathrm{m}$ 相变老化从材料表面向 基体内部传播的主要路径。

\section{3 低温老化动力学}

低温老化会导致氧化锆力学性能下降, 甚至引 起突发失效，从而缩短服役寿命 ${ }^{[24-25]}$ 。因此，为评估 氧化锆在临床应用中的长期稳定性和服役寿命, 需 掌握氧化锆低温老化动力学规律。

在 $37{ }^{\circ} \mathrm{C}$ 的人体内氧化锆陶瓷老化速度慢, 在体 内研究评估周期长, 所以一般采用体外水热老化, 用

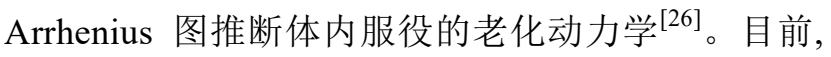
大多数老化动力学通过物相分析 (X-ray diffraction, $\mathrm{XRD}$ )测量的 $\mathrm{m}-\mathrm{ZrO}_{2}$ 相含量与老化时间的变化关系, 并根据 Mehl-Avrami-Johnson (MAJ) 函数进行拟合 [27-28]获得, 可描述为:

$$
f=1-\exp \left(-(b t)^{n}\right)
$$

其中, $f$ 为 $\mathrm{m}-\mathrm{ZrO}_{2}$ 相百分含量, $n$ 取值在 $0.3 \sim 3.5$ (与 材料成核和生长条件相关), $b$ 为激活能, 可表述为:

$$
b=b_{0} \exp \left(-\frac{Q}{R T}\right)
$$

$R$ 为理想气体常数, $Q$ 为老化活化能。基于 $3 \mathrm{~mol} \%$ 氧
化钎稳定四方氧化锆(3Y-TZP)的老化活化能

(106 $\mathrm{kJ} / \mathrm{mol}$ ), Chevalier 等 ${ }^{[29]}$ 计算出: 在 $134{ }^{\circ} \mathrm{C} / 200 \mathrm{kPa}$ 蒸汽 中老化处理 $1 \mathrm{~h}$, 理论上等于 $37{ }^{\circ} \mathrm{C}$ 下进行 3 4 年的 体内时效, 目前 Chevalier 的观点被普遍接受。也有研 究表明加速老化 $1 \mathrm{~h}$ 只相当于人体内服役 1 年 ${ }^{[30]}$ 。

为明确老化动力学中晶粒尺寸、四方相、立方 相与参数 $n 、 Q 、 V_{\max }$ 和 $b_{0}$ 的关系, Wei 等 ${ }^{[31]}$ 采用分 离和量化的方法, 发现立方相的含量和晶粒尺寸与 $V_{\text {max }}$ 呈线性关系，四方相中钇含量和晶粒尺寸与 $b_{0}$ 密切相关, $n$ (或 $Q$ )值与微观结构无关。然而, 由于 MAJ 理论唯象学性质, 在估算氧化锆老化成核-生 长的速率误差较大。Zhigachev 等 ${ }^{[32]}$ 采用解析法建 立了成核-生长理论模型, $R_{\mathrm{N}}$ 为 $\mathrm{m}-\mathrm{ZrO}_{2}$ 的成核率、 $R_{\mathrm{D}}$ 和 $L_{0}$ 为晶核初始直径和深度, $R_{\mathrm{D}}$ 和 $R_{\mathrm{L}}$ 为直径和 深度的生长速率等参数, 理论模型如图 3 所示。

从模型推导出老化动力学的解析表达式, 如下 所示:

$$
\begin{gathered}
F_{\mathrm{m}}(t, 0)=1-\exp \left[-\frac{\pi}{2} N_{\mathrm{r}}\left(\frac{1}{3} R_{\mathrm{D}}^{2} t^{3}+R_{\mathrm{D}} D_{0} t^{2}+\frac{1}{3} D_{0}^{2} t\right)\right] \\
f_{\mathrm{m}}(t)=\frac{1}{3} F_{\mathrm{m}}(t, 0) \times\left(L_{0}+0.5 R_{\mathrm{L}} t\right)
\end{gathered}
$$

发现理论计算值与实验数据的拟合度更高, 这对于 解释在潮湿环境中 $\mathrm{ZrO}_{2}$ 陶瓷的老化动力学, 预测齿 科氧化锆临床应用的服役寿命极其重要。

上述研究描述了氧化锆相变含量随时间变化的 老化动力学, 但并没有明确相变老化向材料内部传 播的过程。目前, 关于相变老化是线性还是非线性 仍存在争论, 众多研究认为老化相变传播为线性, 并对老化前期中 MAJ 模型的相变含量变化形状提 出了质疑。Zhang 等 ${ }^{[33]}$ 采用 $\mu$-Raman 技术研究了 3Y-TZP 的老化动力学, 发现由表面向内部的相变 深度与时间呈线性关系(图 4)。与常规 XRD 相变分 析获取的老化动力学相比, 相变深度传播的线性动

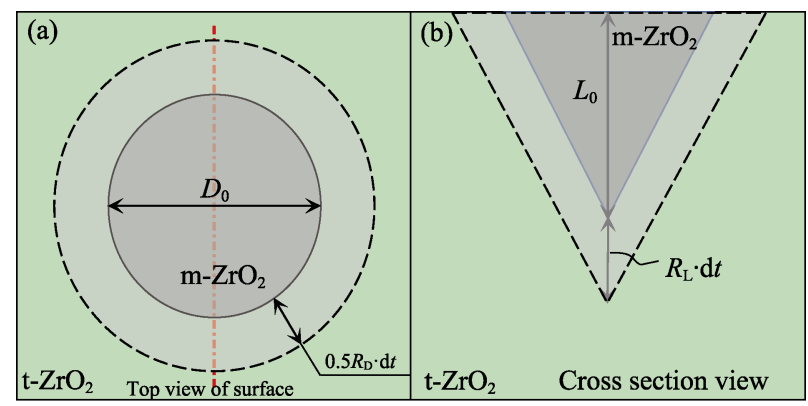

图 $3 \mathrm{~m}-\mathrm{ZrO}_{2}$ 晶核的初始尺寸和生长速率理论模型 ${ }^{[32]}$

Fig. 3 Theoretical model of initial dimensions and growth rate of $\mathrm{m}-\mathrm{ZrO}_{2}$ nuclei $^{[32]}$

(a) Top view of surface; (b) Cross section view 

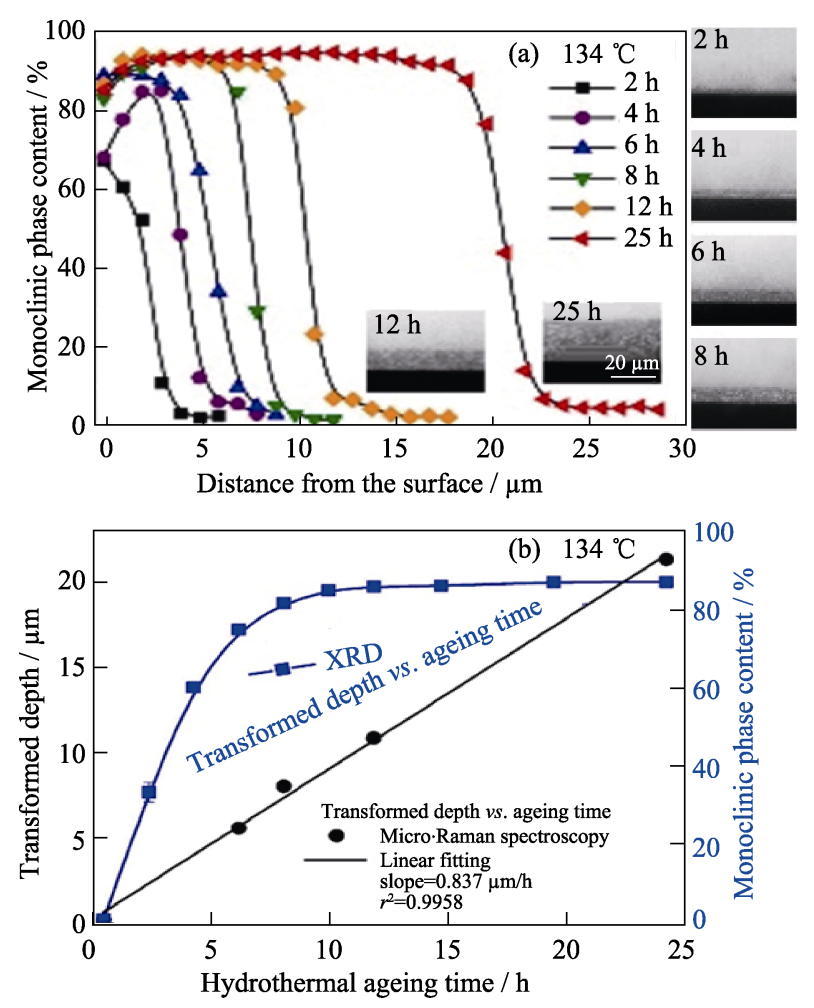

图 $4 \mu$-Raman 分析 3Y-TZP 陶瓷老化动力学 ${ }^{[33]}$

Fig. 4 Ageing kinetics analysis with 3 Y-TZP by $\mu$-Raman spectroscopy ${ }^{[33]}$

(a) $m-\mathrm{ZrO}_{2}$ content depth and corresponding optical images; (b)Transformed depth varies with ageing time

力学更精确地计算出氧化锆老化的活化能, 更直观 和准确地预测 $3 \mathrm{Y}-\mathrm{TZP}$ 在人体内的服役寿命。

\section{2 低温老化表征技术}

随着材料表征技术的不断发展，除常规的 XRD、 扫描电镜( SEM)、原子力显微镜(AFM)、拉曼光谱 (Raman)技术外, 光学干涉(Optical interference, OI)、 光学相干断层扫描(Optical coherence tomography, OCT)、聚焦离子束(Focused ion beam, FIB)等新型表 征技术的发展和成熟, 实现了从原子级到宏观上监 测老化过程中相含量、微观形貌、结构性能的动态 变化, 下面介绍了表征相含量和结构形貌各自的技 术特点并进行详细比较。

\section{1 相含量分析}

氧化锆 LTD 本质是 $\mathrm{t}-\mathrm{m}$ 马氏体相变, 其过程很 容易采用对晶体结构变化敏感的技术表征方法, 如 $\mathrm{XRD}$ 常用来检测氧化锆老化后 $\mathrm{m}-\mathrm{ZrO}_{2}$ 含量。 $\mathrm{m}-\mathrm{ZrO}_{2}$ 含量计算方法是基于 Garvie 和 Nicholson ${ }^{[34]}$ 提出并被 Toraya 等 ${ }^{[35]}$ 修正的公式:

$$
X_{\mathrm{m}}=\frac{I_{\mathrm{m}}\{\overline{1} 11\}+I_{\mathrm{m}}\{111\}}{I_{\mathrm{m}}\{\overline{1} 11\}+I_{\mathrm{m}}\{11\}+I_{\mathrm{t}}\{101\}}
$$

$\mathrm{XRD}$ 技术不仅能够检测样品的近表面 $\mathrm{m}$ 相含 量, 而且相变深度为几微米 $(<5 \mu \mathrm{m})$ 时, 可以通过改 变入射角大小测量单斜相含量推算出相变深度, Koyama 等 ${ }^{[36]}$ 通过改变 $X$ 射线入射角 $\omega$ 大小测量单 斜相含量, 研究发现随着入射角增大, $\mathrm{m}$ 相峰强度 减弱, 如图 5 所示, 证实了 3Y-TZP 的 t-m 相变老化 首先从材料表面开始，然后向基体内部传播。 Gremillard 等 ${ }^{[37]}$ 利用拟合方程和拉普拉斯变换等数 学工具, 发现可检测相变深度的范围对 X 射线穿透 深度及拟合方程的影响。

其次, Raman 技术也是表征氧化锆陶瓷的最有 力技术之一。Raman 技术 (微探针和共焦结构)对单 斜相含量变化比 XRD 更敏感, 还可区分 $\mathrm{t}$ 相和 $\mathrm{t}^{\prime}$ 相 ( $\mathrm{t}$ 相快速冷却形成的立方晶相 $\left.\mathrm{t}^{\prime}\right)$; 空间分辨率高, 对 $\mathrm{t}-\mathrm{m}$ 相变局部区更敏感。目前, 有三种函数关系 表达式: 线性、幂数、对数来计算单斜相含量, 如表 1 所示。此外, 利用 Raman 光谱峰位移来检测应变 导致的原子间距离的变化, 可以计算出材料的应力 大小。对于配有共聚焦结构的 $\mu$-Raman 技术, 可以 表征单斜相的含量和三维应力状态。

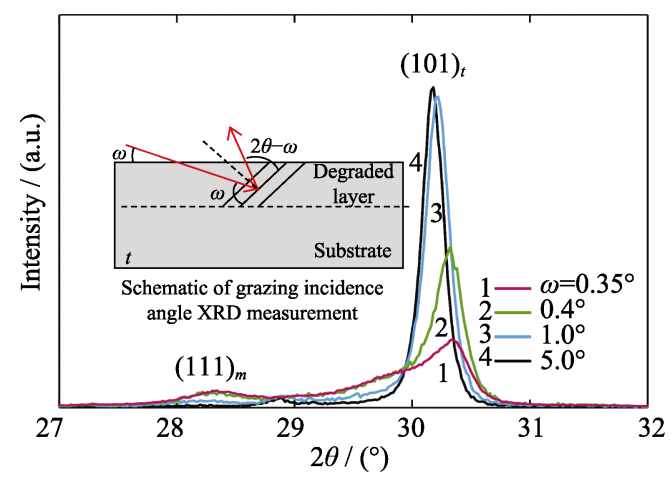

图 5 老化后 $3 \mathrm{Y}-\mathrm{TZP}$ 不同入射角的 XRD 图谱 ${ }^{[37]}$

Fig. 5 XRD profiles for different incidence angles $\omega$ in the surface of the 3 Y-TZP specimen after LTD test ${ }^{[37]}$

表 1 基于拉曼光谱的单斜相定量分析模型

Table 1 Proposed models for monoclinic phase quantification by Raman spectroscopy

\begin{tabular}{|c|c|}
\hline & Equation \\
\hline \multirow{2}{*}{ Linear } & $V_{\mathrm{m}}=\frac{I_{\mathrm{m}}^{181}+I_{\mathrm{m}}^{190}}{k\left(I_{\mathrm{t}}^{147}+\delta I_{\mathrm{t}}^{265}\right)+I_{\mathrm{m}}^{181}+I_{\mathrm{m}}^{190}}$ \\
\hline & $\begin{array}{lc}\text { Clarke and Adar }{ }^{[38]} & \delta=1 ; k=0.97 \\
\text { Katagiri, et al. }{ }^{[39]} & \delta=0 ; k=2.2 \pm 0.2 \\
\text { Lim, et al. }{ }^{[40]} & \delta=1 ; k=0.33 \pm 0.33\end{array}$ \\
\hline Power law ${ }^{[41]}$ & $V_{\mathrm{m}}=\sqrt{0.19-\frac{0.13}{\frac{I_{\mathrm{m}}^{181}+I_{\mathrm{m}}^{190}}{I_{\mathrm{t}}^{147}+I_{\mathrm{m}}^{181}+I_{\mathrm{m}}^{190}}-1.01}}-0.56$ \\
\hline Logarithmic $^{[42]}$ & $V_{\mathrm{m}}=0.65+0.39 \lg \left(\frac{I_{\mathrm{m}}^{181}+I_{\mathrm{m}}^{190}}{I_{\mathrm{t}}^{147}+I_{\mathrm{t}}^{265}+I_{\mathrm{m}}^{181}+I_{\mathrm{m}}^{190}}\right)$ \\
\hline
\end{tabular}




\section{2 微观形貌表征}

对于两种无损表面形貌监测技术, 如 OI 和 AFM, 对材料老化初期表面形貌变化敏感, 通过图 像分析技术计算出表面的单斜相含量及粗䊁度, 还 能确定和区分老化初期的相变区，但都无法获得基 体内部的相变信息。AFM 空间分辨率更高(原子分 辨率), 可以清楚监测老化早期的形核-生长过程。 因景深度和可视区域限制，难以检测齿科修复体咬 合面等非平面样品, 可定性分析而不能定量统计分 析，而 OI 技术空间尺度为微米级，能够检测老化初 期的单斜相数目及大小来推算老化动力学。

上述技术仅提供表面或近表面相变信息，SEM 技术却可以检测基体内部相变信息，直接评估 $\mathrm{t}-\mathrm{m}$ 相变深度及推算老化动力学, 但检测技术会破坏样 品的完整性。与 SEM 相比, OCT 光学相干断层分析 不仅属于无损检测技术，还同时简化了样品制备步 骤并缩短了检测时间。随着 FIB 技术的出现，人们 探明了老化后氧化锆陶瓷力学性能劣化主要是由于 微裂纹造成的, 与 $\mathrm{m}-\mathrm{ZrO}_{2}$ 相含量的变化几乎无关。

\section{3 低温老化的影响因素}

为理解 t-m 相变所涉及的因素, Lange 等 ${ }^{[43]}$ 首次 描述了氧化锆 $\mathrm{t}-\mathrm{m}$ 相变的热力学, 相变总自由能的 变化如下:

$$
\Delta G_{\mathrm{t}-\mathrm{m}}=\Delta G_{\mathrm{c}}+\Delta G_{\mathrm{se}}+\Delta G_{\mathrm{s}}
$$

式中 $\Delta G_{\mathrm{c}}$ 为化学自由能变化 (取决于温度和成分、稳 定剂含量及类型), $\Delta G_{\mathrm{se}}$ 为应变自由能变化(取决于 基体模量、相变后晶粒尺寸及形状、应力情况), $\Delta G_{\mathrm{s}}$ 为表面自由能变化(取决于新界面和微裂纹)。基于 相变过程中自由能变化情况, 下面重点讨论了影响 低温老化的主要因素, 即稳定剂、残余应力、晶粒 尺寸。

\section{1 稳定剂}

理论上所有的稳定剂都可用以辅助制备 TZP 材 料, 但在 TZP 材料中 $\mathrm{Y}_{2} \mathrm{O}_{3}$ 稳定剂应用最广泛。与 其它稳定剂一样, 其特点是向 $\mathrm{ZrO}_{2}$ 晶格引入 $\mathrm{Y}_{2} \mathrm{O}_{3}$, 为保持电荷平衡而产生氧空位, 从而起到稳定 $\mathrm{t}$ 相 氧化锆的作用。通常加入 $3 \mathrm{~mol} \%$ 的 $\mathrm{Y}_{2} \mathrm{O}_{3}$ 稳定剂到 氧化锆中, 在室温下获得稳定存在的 $3 \mathrm{Y}-\mathrm{TZP}$ 陶瓷。

众所周知, Y-TZP 在潮湿环境中易发生低温老 化现象，通过增加稳定剂 $\mathrm{Y}_{2} \mathrm{O}_{3}$ 含量可以有效抑制 低温老化, 提高材料的服役性能。但随着稳定剂含 量增加, 四方相含量减少且稳定性提高, 不利于材 料发生 $\mathrm{t}-\mathrm{m}$ 相变增韧, 从而导致力学性能下降。由 此可见, 仅用 $\mathrm{Y}_{2} \mathrm{O}_{3}$ 作为稳定剂的氧化锆陶瓷综合
性能受限。采用 $\mathrm{CeO}_{2} 、 \mathrm{Yb}_{2} \mathrm{O}_{3}$ 等类型稳定剂[26]替换 $\mathrm{Y}_{2} \mathrm{O}_{3}$ 稳定剂或混合两种或多种稳定剂代替单一 $\mathrm{Y}_{2} \mathrm{O}_{3}$ 稳定剂的实验研究发现, 对氧化锆 LTD 有良好 的抑制作用。

\section{2 残余应力}

氧化锆老化过程中伴随 $\mathrm{t}-\mathrm{m}$ 相变, 这与应力诱 导相变有直接联系。氧化锆中四方相处于稳定的应 力状态下, 当应力状态发生改变时, 四方相会向单 斜相转变发生低温老化现象。当晶粒处于拉伸状态 时, 为减小初始的拉伸状态, 通过转化为 $\mathrm{m}-\mathrm{ZrO}_{2}$ 扩 大其尺寸而达到重新平衡状态, 这种现象可以根据 勒夏特列原理解释。

其次，应力是直观可见的，齿科氧化锆的咬合 面具有复杂几何形状而难以精确计算, 需要考虑义 齿截面积下限，避免增加局部应力而导致材料服役 失效。应力对氧化锆低温老化的影响需考虑三种类 型：残余应力(加工和抛光产生的应力)、外加应力和 $\mathrm{t}-\mathrm{m}$ 相变产生的应力, 通常相变应力和残余应力比 外加应力更大。有研究发现材料中残余应力的大小 和形式会直接影响氧化锆的老化行为, 但与氧化锆 陶瓷的微观结构和表面处理工艺也有关系，这说明 残余应力不是决定材料老化敏感性的唯一因素。

\section{3 晶粒尺寸}

对于多晶材料的相稳定性存在晶粒尺寸(Grain size, GS)效应，这是由界面能对体系总自由能的贡 献所决定的。对于 Y-TZP 陶瓷，由于 GS 临界值的 定义不够明确，即在一定温度下会发生 $\mathrm{t}-\mathrm{m}$ 相变。 虽然将晶粒尺寸作为单独的因素讨论, 但氧化锆 $\mathrm{t}-\mathrm{m}$ 相变的晶粒尺寸临界值受到许多因素影响, 如 材料的化学成分、稳定剂类型和含量、复杂的残余 应力条件等。

通过热力学理论可知: 当细化晶粒到小于相变临 界值的晶粒尺寸时，将有效抑制低温老化现象的发 生 ${ }^{[19]}$ 。目前, 研究报道的 Y-TZP 陶瓷(特别是高致密性 陶瓷)的 GS 临界值的分布范围很广, 在 $50 \sim 500 \mathrm{~nm}^{[44]}$; 此外, Y-TZP 陶瓷在不同温度下具有不同的 GS 临界 值。对于处于热力学稳定状态的 $\mathrm{t}-\mathrm{ZrO}_{2}$ 不会发生相 变, 但当系统状态发生改变时, 触发 $t-m$ 相变从而 降低材料的服役性能。

\section{4 抑制 Y-TZP 老化的方法}

氧化锆陶瓷的 LTD 与晶粒尺寸、稳定剂、残余 应力大小有关, 因此现已从氧化锆陶瓷的微观结 构、制备工艺、开发设计 3 方面解决其存在的 LTD 问题。 


\section{1 控制材料微观结构}

晶粒尺寸与 $\mathrm{t}-\mathrm{ZrO}_{2}$ 陶瓷的稳定性紧密相关, 因 而决定氧化锆陶瓷的 $\mathrm{t}-\mathrm{m}$ 相变增韧和老化行为。基 于此, $\mathrm{Lu}$ 和 Chen 等 ${ }^{[44]}$ 描述了氧化锆 $t-m$ 相变老化行 为与晶粒尺寸的关系图, 推导出氧化锆在空气和水 中相变临界尺寸分别为 0.52 和 $0.37 \mu \mathrm{m}$ 。结果说明: 在热水或水蒸气条件下, 氧化锆更容易发生老化。

Hallmann 等 ${ }^{[45]}$ 发现: 为使 Y-TZP 材料具有良好的 抗老化能力, 氧化锆晶粒尺寸应该控制在 $0.3 \sim 0.4 \mu \mathrm{m}$ 。 烧结温度控制在 $1300{ }^{\circ} \mathrm{C}$ 、晶粒尺寸小于 $0.3 \mu \mathrm{m}$ 时, 老化后并未发现 $\mathrm{m}$ 相、结构破坏、晶粒拔出等现象; 当烧结温度高于 $1500{ }^{\circ} \mathrm{C}$ 时, 晶粒尺寸大于 $0.3 \mu \mathrm{m}$, 随着晶粒尺寸变大, 材料表面结构有明显的破坏 (图 6)。Xiong 等 ${ }^{[46]}$ 通过在纳米尺度上控制晶粒尺寸 的变化, 制备出平均粒径约为 $87 \mathrm{~nm}$ 的 $3 \mathrm{Y}-\mathrm{TZP}$ 纳米 陶瓷, 在 $134{ }^{\circ} \mathrm{C} / 2 \times 10^{5} \mathrm{~Pa}$ 条件下经过时效处理 $100 \mathrm{~h}$, 发现不存在明显的低温老化现象, 原因是 3Y-TZP 纳米陶瓷不仅具有高致密性, 而且晶粒尺寸远小于 临界相变尺寸。

\section{2 优化材料制备工艺}

粉末的制备、坏体成型烧结、烧结体后处理等 工艺环节直接影响氧化锆陶瓷抗老化性能。因此, 研究人员通过优化和改进制备工艺来抑制 LTD, 提 高其在临床应用中的稳定性和可靠性。

\subsection{1 粉体制备工艺}

$\mathrm{Swab}^{[47]}$ 研究发现氧化锆老化行为不仅与烧结 方法和晶粒尺寸相关, 还取决于初始粉末的性能。 Lawson 等 ${ }^{[8]}$ 发现采用不同的方法制备的 3Y-TZP 粉 末, 其抗低温老化能力存在明显差异。虽然, 共沉淀 法和气相反应-等离子包覆法已应用于 Y-TZP 粉末 的制备, 但制备的粉末因粒径及稳定剂 Yttria 分布 不均匀易发生 t-m 相变老化。

相比于传统球磨混合方法, Deville 等 ${ }^{[49]}$ 采用溶 胶法制备的氧化锆粉末无团聚现象, 烧结体具有较 小的晶粒尺寸和良好的致密性。Smirnov ${ }^{[50]}$ 证实了 粉末合成方法在 Y-TZP 陶瓷 LTD 行为中扮演重要
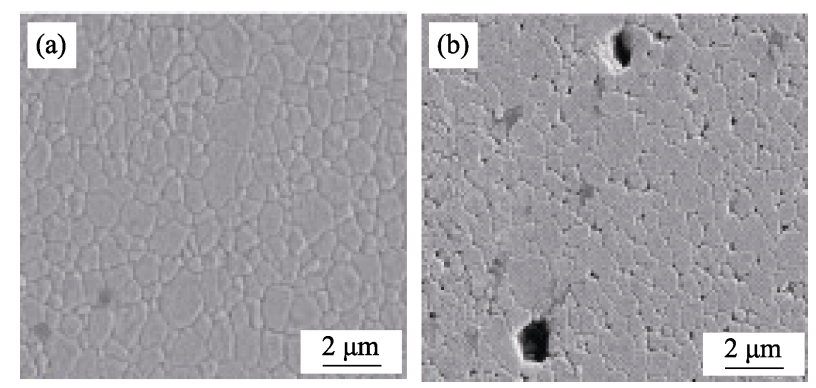

图 6 Y-TZP 陶瓷微观形貌 ${ }^{[45]}$

Fig. 6 SEM images of the Y-TZP ceramic ${ }^{[45]}$

(a) before and (b) after LTD
角色, 研究发现采用 $\mathrm{CO}_{2}$-LAVA 法和湿法混合制备 的 2Y-TZP 纳米粉体，经过等离子烧结后, $2 \mathrm{Y}-\mathrm{TZP}$ 陶 瓷具有明显的抗 LTD 性能和高断裂韧性 $\left(13 \mathrm{MPa} \cdot \mathrm{m}^{0.5}\right)$, 归因于晶粒尺寸和 Yttria 分布(图 7)。

\subsection{2 陶瓷制备工艺}

实际应用中, 由于晶粒是相互限制的, 很难自 由增大或减小。在烧结过程中, 晶粒尺寸会随烧结 温度升高和保温时间延长而增大，导致晶粒粗化而 加速老化。为避免晶粒粗化并保证致密性, 人们探 讨了不同烧结技术如何有效地抑制低温老化的发生。

Sutharsini 等 ${ }^{[11]}$ 等采用两步烧结(TSS)工艺制备 Y-TZP 陶瓷, 发现随着保温时间的延长, 在不引起 晶粒粗化的情况下, 能有效地促进致密化。样品的 相对密度和晶粒尺寸分别为 $97 \%$ 和 $0.25 \mu \mathrm{m}$, 烧结 的样品力学性能不受烧结时间的影响, 表现出优异 的耐低温老化性能。在缩短烧结时间和降低烧结温 度的情况下, Presenda 等 ${ }^{[52]}$ 采用微波烧结(MW)技术 制备的氧化锆陶瓷致密性高, 且 $\mathrm{c}$ 相含量降低, 样 品在水热条件下暴露处理 $200 \mathrm{~h}$, 几乎没有 $\mathrm{m}$ 相产 生, 表现出良好的抗水热老化性能。Chintapalli 等 ${ }^{[53]}$ 采用等离子烧结(SPS)技术获得小晶粒尺寸的陶瓷, 晶粒边缘呈圆形，降低了晶界交汇处的残余应力， 提高了相变势垒 $\Delta \mathrm{G}_{\mathrm{t}-\mathrm{m}}$ 从而抑制 LTD。此外发现, 孔 隙率对样品耐水热老化性能无明显影响。
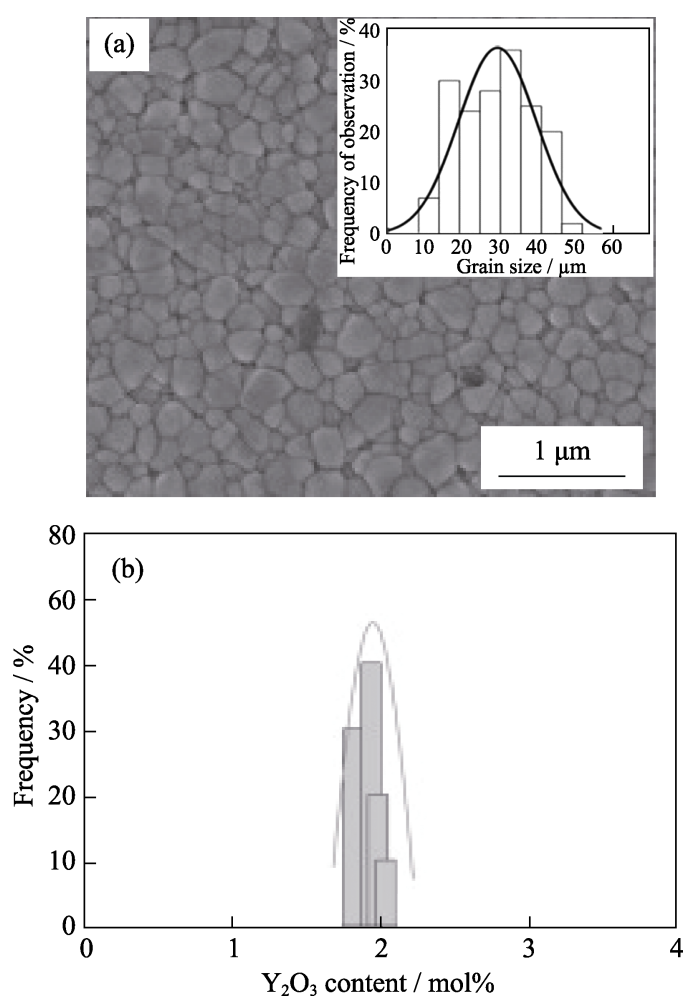

图 7 2Y-TZP 陶瓷晶粒尺寸 ${ }^{[50]}$

Fig. 7 2Y-TZP ceramic ${ }^{[50]}$

(a) SEM images together with the corresponding grain size distribution, and (b) semi-quantitative $\mathrm{Y}_{2} \mathrm{O}_{3}$ distribution 


\subsection{3 陶瓷后处理工艺}

$\mathrm{t}-\mathrm{m}$ 相变老化开始于材料表面，研究人员自然 想到对氧化锆表面进行处理, 从而阻止 LTD 向基体 内部渗透，最终保持氧化锆的整体机械强度 ${ }^{[54]}$ 。

Deville 等 ${ }^{[55]}$ 采用不同形式的抛光手段研究表 面残余应力对 Y-TZP 陶瓷 LTD 的影响。他们发现, 相比于精抛, 粗抛可以抑制 LTD, 原因是粗抛表面 形成压缩应力, 而精抛后划痕周围产生拉应力, 容 易触发 t-m 相变。Inokoshi 等 ${ }^{[56]}$ 研究表明 3Y-TZP 经 过粗抛、喷砂处理后, 表面会产生残余压力层, 材料 的抗低温老化能力得到提高。然而, 对 $\mathrm{Al}_{2} \mathrm{O}_{3} / 3 \mathrm{Y}-\mathrm{TZP}$ 粗抛后发现, 材料的抗低温老化能力略微降低, 原 因是粗抛后材料 $\mathrm{c}-\mathrm{ZrO}_{2}$ 含量降低, 进而影响到材料 的抗低温老化能力。由此得出, 残余应力的等级和大 小不是决定材料的抗降解敏感性的唯一因素。Wei 等 ${ }^{[57]}$ 研究了外加载荷对氧化锆老化敏感性的影响, 发现无论是拉应力还是压应力均会加速氧化锆的低 温老化。

除了改善表面处理工艺外, 利用化学反应在表 面形成保护层也是一种减缓、抑制老化的措施。 Giles 等 ${ }^{[58]}$ 首次报道了氮气处理氧化锆陶瓷可以提 高抗老化性能，原因是 $\mathrm{N}$ 离子在氧化锆晶格中扩散 而占据氧位, 产生氧空位而提高抗 LTD 能力。Chung 等 ${ }^{[59]}$ 在 $\mathrm{ZrN}$ 粉末床中烧结 3Y-TZP 材料, 发现其表 面层由稳定的 $\mathrm{t}^{\prime}$ 相和 $\mathrm{c}$ 相构成; 避免水分子与基体 内 $\mathrm{t}$ 相接触, 延缓了 LTD 向基体内部传播, 同时降 低了材料表面层的韧性和应用可靠性。为避免材料 表面力学性能的退化, Valle 等 ${ }^{[00]}$ 通过优化渗氮工艺
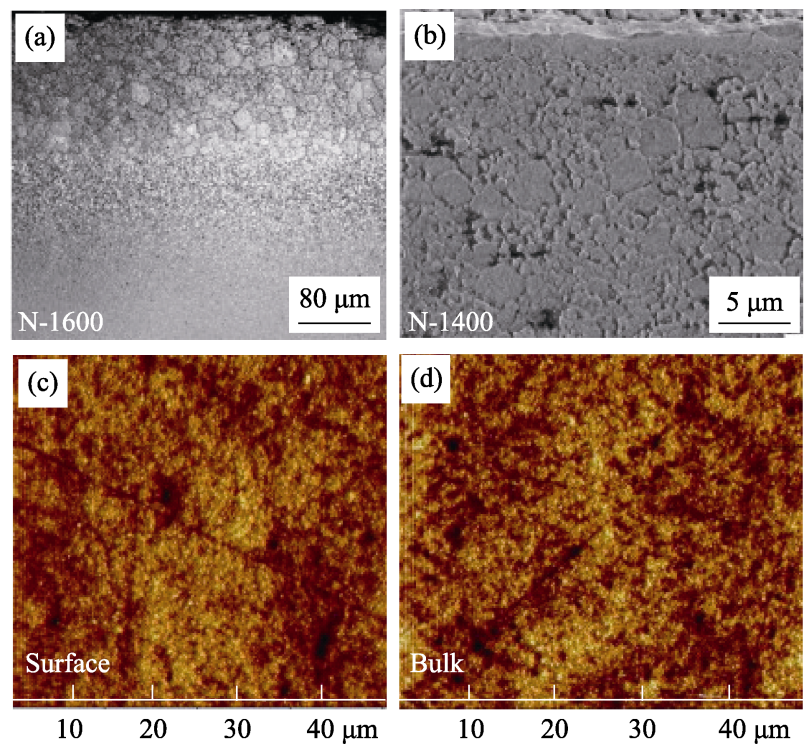

图 8 表面氮处理的样品老化后形貌 ${ }^{[60]}$

Fig. 8 Morphologies of samples treated with surface nitrogen after aging ${ }^{[60]}$

(a) LSCM image of N-1600; (b) SEM image of N-1400; AFM images of N-1400 (c) surface and (d) bulk
条件, 得出 $\mathrm{ZrN}$ 粉末渗氮最佳工艺条件为 $1400{ }^{\circ} \mathrm{C}$ 下保温 $1 \mathrm{~h}, 3 \mathrm{Y}-\mathrm{TZP}$ 微观结构变化最小, 避免出现 $\mathrm{t}^{\prime}$ 相和 $\mathrm{c}$ 相, 水热老化实验后样品表面和基体形貌有 明显变化(图 8)。此外, 通过表面镀层工艺也可以提 高氧化锆陶瓷的抗老化性能, Hübsch 等 ${ }^{[61]}$ 采用物理 气相沉积 $(\mathrm{PVD})$ 技术, 将钛氧化物 $\left(\mathrm{Ti}_{x} \mathrm{O}_{y}\right)$ 及钛氧化 物-氧化铝 $\left(\mathrm{Ti}_{x} \mathrm{O}_{y}-\mathrm{Al}_{x} \mathrm{O}_{y}-\mathrm{Ti}_{x} \mathrm{O}_{y}\right)$ 透明薄膜沉积在氧化 锆基陶瓷表面, 结果表明涂层的出现减缓了 $\mathrm{t}-\mathrm{m}$ 相 变的进行，从而抑制老化现象的发生。

\section{3 开发设计复合陶瓷}

控制材料的微观结构, 优化制备工艺等方法可 以提高氧化锆的抗老化性能，但都会降低氧化锆的 力学性能, 因此研究人员通过开发设计氧化锆复合 陶瓷来抑制 LTD 行为, 如添加第二相颗粒。

\subsection{1 添加氧化物}

以往文献研究表明 Y-TZP 掺杂具有广泛的应用 前景，如 $\mathrm{CuO} 、 \mathrm{MgO} 、 \mathrm{TiO}_{2} 、 \mathrm{Fe}_{2} \mathrm{O}_{3} 、 \mathrm{MnO}_{2}$ 等氧化 物掺杂剂已经获得研究和应用 ${ }^{[45,62-65]}$, 但只有少数 掺杂剂在抗老化性能和力学性能之间达到平衡。已 知的含有或添加 $0.25 \mathrm{wt} \% \mathrm{Al}_{2} \mathrm{O}_{3}$ 的 $3 \mathrm{Y}-\mathrm{TZP}$ 粉末具有 优异的力学性能和抗 LTD 性能, 在齿科修复领域中 被广泛应用。原因可能为 $\mathrm{Al}_{2} \mathrm{O}_{3}$ 的加入提高了基体 的杨氏模量和应变自由能, 从而提高了四方相的稳 定性; $\mathrm{Al}_{2} \mathrm{O}_{3}$ 比 $\mathrm{Y}_{2} \mathrm{O}_{3}$ 更易水解, 在材料表面优先形成 $\mathrm{Al}(\mathrm{OH})_{3}$ 而阻碍相变向基体内部渗透。

然而, 氧化铝改善 3Y-TZP 老化性能的机理尚 未明确, 有研究认为当掺杂金属的阳离子半径大于 或小于 $\mathrm{Zr}^{4+}$ 离子时, 在偏析驱动力作用下掺杂阳离 子会在晶界处偏析 ${ }^{[66-68]}$ 。掺杂后为保持电荷平衡会 在晶界处产生氧空位, 偏析的阳离子与氧空位相结 合, 从而打断 $\mathrm{OH}^{-}$扩散造成氧空位的耗散, 有效抑 制 LTD。Zhang 等 ${ }^{[69]}$ 添加不同含量 $\mathrm{Al}_{2} \mathrm{O}_{3}$, 研究其对 3Y-TZP 抗低温老化性能的影响, 发现 $\mathrm{Al}_{2} \mathrm{O}_{3}$ 在氧化 锆中存在溶解极限, 过量的 $\mathrm{Al}_{2} \mathrm{O}_{3}$ 可能会引起残余 应力, 从而提高低温老化降解速率; 结果表明添加 量为 $0.25 \mathrm{wt} \%$ 时抑制效果最优, 晶界处 $\mathrm{Al}^{3+}$ 的溶解 和偏析有效抑制了 Y-TZP 老化, 偏析的 $\mathrm{Al}^{3+}$ 在晶界 处均匀分布，如图 9 所示。
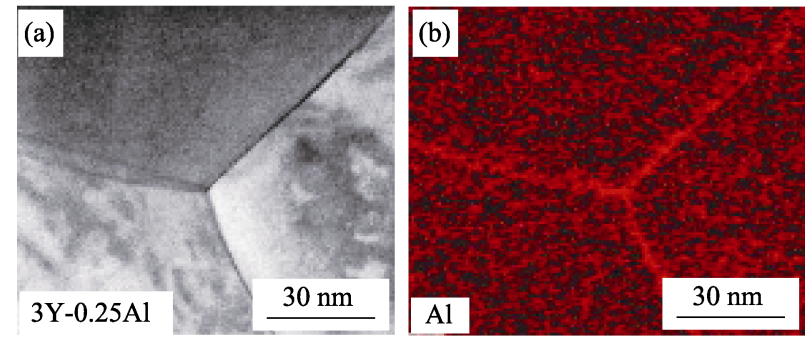

图 $93 \mathrm{Y}-0.25 \mathrm{Al}$ 陶瓷 ${ }^{69]}$

Fig. 9 3Y-0.25 Al ceramic ${ }^{[69]}$

(a) TEM image of grain boundaries; (b) Corresponding Al elemental map 
氧化铝的添加会影响 3Y-TZP 陶瓷的半透明性, 从而需控制氧化铝的添加量以制造高半透明性的齿 科氧化锆陶瓷 ${ }^{[70]}$ 。Zhang 等 ${ }^{[71]}$ 通过掺杂阳离子半径 不同于锆离子的氧化物来改善材料的抗 LTD 能力。 结果发现, 掺杂偏析是制备水热稳定、高半透明性 材料的关键, 采用半径过大的阳离子具有明显的晶 界偏析; $0.2 \mathrm{~mol} \% \mathrm{La}_{2} \mathrm{O}_{3}$ 和 $0.1 \sim 0.25 \mathrm{wt} \% \mathrm{Al}_{2} \mathrm{O}_{3}$ 为最 佳掺杂量, 材料具有优异的力学性能和水热稳定性, 同时半透明性提高了 $42 \%$ 。为制备水热稳定、高半 透明性, 同时保持优异力学性能的齿科 3Y-TZP 陶 瓷提供了实验依据。

另一种有潜力的氧化物掺杂剂是 $\mathrm{SiO}_{2}{ }^{[72]}$, 这是 因为 $\mathrm{SiO}_{2}$ 削弱了三晶交汇处的应力, 减缓了应力诱 导 $\mathrm{t}-\mathrm{m}$ 转变, Gremillard 等 ${ }^{[73]}$ 研究证实了该结论, 并 发现分布在氧化锆晶界结合处的非晶相 $\mathrm{SiO}_{2}$ 减少 了三晶交汇处应力的集中效应。此外，非晶相 $\mathrm{SiO}_{2}$ 削弱了 $\mathrm{t}-\mathrm{m}$ 相变形成的尖端应力, 避免了尖端应力 诱导单斜相的形核和生长，从而阻碍 $\mathrm{t}-\mathrm{m}$ 相变向基 体内部传播。Samodurova 等 ${ }^{[74]}$ 发现 $\mathrm{Al}_{2} \mathrm{O}_{3}$ 和 $\mathrm{SiO}_{2}$ 的共掺杂对水诱导相变的成核和传播都有抑制作用, 其具体的作用方式和机制，如图 10 所示; 圆形晶粒 的晶界处伴有 $\mathrm{SiO}_{2}$ 玻璃相, 降低内应力来提高应变 自由能; 此外, $\mathrm{Al}_{2} \mathrm{O}_{3}$ 的断裂方式主要表现为穿晶断 裂, 说明强烈的晶界结合力限制了转化层微裂纹的 扩展，最终抑制 LTD 的发生和传播。

\subsection{2 添加非氧化物}

Mohamed 等 ${ }^{[75]}$ 发现碳纳米管结构(CNTs)添加 到 $3 \mathrm{Y}-\mathrm{TZP}$ 基体中, $3 \mathrm{Y}-\mathrm{TZP} / \mathrm{CNTs}$ 复合陶瓷应变自由 能增加, 随之 $\mathrm{t}-\mathrm{m}$ 相变势垒增加, 从而抑制 LTD, 并 且无毒副作用。为深入解释添加碳纳米管的作用机 制, Morales 等 ${ }^{[76]}$ 在 3Y-TZP 基体中加入少量的单壁 碳纳米管(SWNTs), 发现晶界处的碳纳米管结构通 过弯曲和晶间滑移吸收了马氏体相变产生的弹性能, 延缓了微裂纹的扩展并提高了抗老化性能。相比超 声探头分散(UP)法，经超声波浴分散(UB)的混合粉 体制备的复合陶瓷碳纳米管分布均匀, 表现出优异 的力学性能和耐老化性能(图 11)。
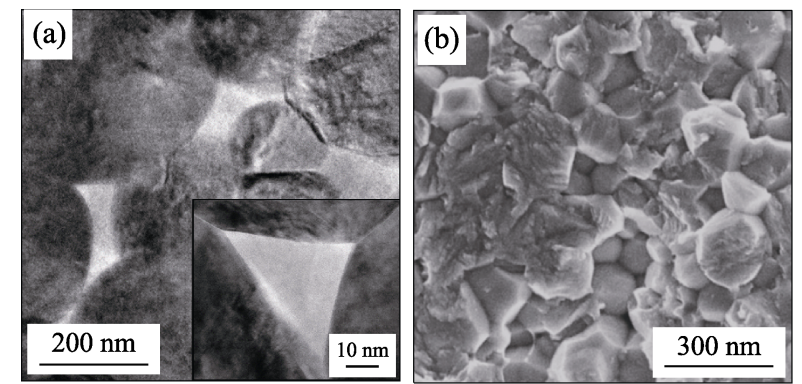

图 $100.05 \mathrm{~A}-\mathrm{TZ} / \mathrm{SiO}_{2}$ 的样品形貌 ${ }^{[74]}$

Fig.10 0.05A-TZ/ $/ \mathrm{SiO}_{2}$ specimen images of fractured surface $\mathrm{e}^{[74]}$ (a) TEM image; (b) SEM image
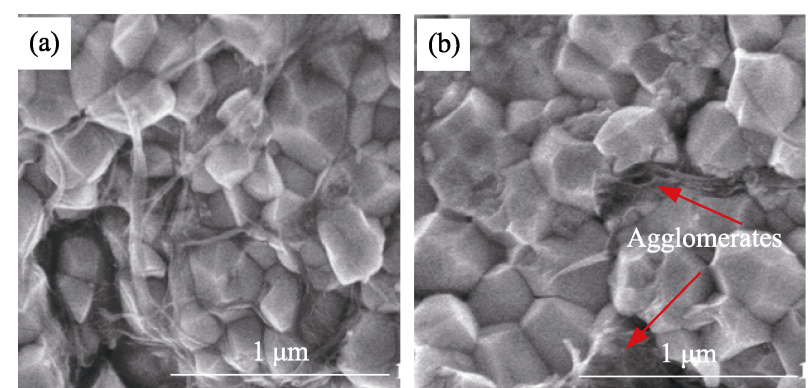

图 $113 \mathrm{Y}-\mathrm{TZP} / \mathrm{SWNT}$ 陶瓷中单壁碳纳米管的分布 ${ }^{[76]}$

Fig. 11 SEM images of the SWNT distribution within the $3 \mathrm{Y}-\mathrm{TZP} / \mathrm{SWNT}$ ceramic $^{[76]}$

(a) $\mathrm{C} 1.5-\mathrm{UB}$; (b) C1.5-UP

\section{5 总结与展望}

在齿科修复领域，Y-TZP 陶瓷凭借优异的力学 性能、良好的生物相容性、色泽自然美观已被广泛 用作核心修复材料。然而，在低温潮湿或口腔环境 中，氧化锆会自发地发生 $\mathrm{t}-\mathrm{m}$ 相变，临床应用的稳 定性显著降低。研究者通过优化制备工艺和调整设 计材料体系来提高综合力学性能并解决其存在的低 温老化问题，使其能在口腔环境中长期稳定地服役。

未来的研究还需在以下方面实现突破:

(1)建立和完善齿科氧化锆低温老化的理论。明 确齿科氧化锆陶瓷的低温老化机制及老化动力学规 律，阐明 $\mathrm{H}_{2} \mathrm{O}$ 的具体作用机制，建立全面系统的抗 LTD 理论, 完善氧化锆在生物体内长期服役的评价 方法。

(2)优化和开发齿科氧化锆制备工艺。传统成形 /磨削工艺会引起表面应力集中和诱导微裂纹产生, 从而容易触发和加速 $\mathrm{t}-\mathrm{m}$ 相变老化。将 $3 \mathrm{D}$ 打印技 术引入到齿科修复领域 ${ }^{[10]}$, 可实现齿科修复设计到 成形的一体化，避免磨削导致的表面损伤问题，还 能满足患者对齿科修复的个性化需求。

(3)开发设计齿科氧化锆复合材料。为满足齿科 材料高强韧性的需求和解决其存在的低温老化问题, 氧化铝的添加不仅提高了氧化锆陶瓷的抗 LTD 性 能, 还能将氧化铝的高硬度与氧化锆的强韧性结合, 对扩大临床应用范围和提高稳定性将具有重要意义。

(4)以临床应用为导向，提高齿科材料的健康功 能化特性。随着功能材料的研究和发展, 非金属矿 物材料因具有独特的理化结构和功能属性被广泛应 用于医疗、保健等领域; 如电气石远红外复合陶瓷 的应用，为开发具有远红外性能、有益于人体健康 的全新齿科材料提供了理论基础和实验依据 ${ }^{[77]}$ 。下 一步还将以临床应用为导向, 进一步提高齿科材料 的健康促进功能化特性。 
义齿是一种具有复杂外形的功能化、个性化的 生物医用修复体，在潮湿的口腔唾液等复杂生化条 件下服役, 长期承受温度波动、 $\mathrm{pH}$ 变化、细菌活性 及频繁的咀嚼载荷 ${ }^{[78]}$ 。当前, 人口老龄化日趋严重, 人们追求美好生活的向往更加强烈。随着齿科氧 化锆陶瓷抗老化性能的提高, 以及健康功能化的 未来需求，氧化锆在生物医用领域的应用将会更加 广泛。

\section{参考文献}

[1] TURON-VINAS M, ANGLADA M. Strength and fracture toughness of zirconia dental ceramics. Dental Materials, 2018, 34(3): 365-375.

[2] FERRARI M, VICHI A, ZARONE F. Zirconia abutments and restorations: from laboratory to clinical investigations. Dental Materials, 2015, 31(3): 63-76.

[3] GAUTAM C, JOYNER J, GAUTAM A, et al. Zirconia based dental ceramics: structure, mechanical properties, biocompatibility and applications. Dalton Transactions, 2016, 45(48): 19194-19215.

[4] GARVIE R C, HANNINK R H, PASCOE R T. Ceramic steel? Nature, 1975, 258(5537): 703-704.

[5] DENRY I, KELLY J R. State of the art of zirconia for dental applications. Dental Materials, 2008, 24(3): 299-307.

[6] TURON-VINAS M, ANGLADA M. Strength and fracture toughness of zirconia dental ceramics. Dental Materials, 2018, 34(3): 365-375.

[7] DENRY I, HOLLOWAY J. Ceramics for dental applications: a review. Materials, 2010, 3(1): 351-368.

[8] NAKONIECZNY D S, ZIĘBOWICZ A, PASZENDA Z K, et al. Trends and perspectives in modification of zirconium oxide for a dental prosthetic applications: a review. Biocybernetics and Biomedical Engineering, 2017, 37(1): 229-245.

[9] LI R W K, CHOW T W, MATINLINNA J P. Ceramic dental biomaterials and CAD/CAM technology: state of the art. Journal of Prosthodontic Research, 2014, 58(4): 208-216.

[10] ZHU DONG-BIN, CHU RUI-QING, ZHANG XIAO-XU, et al. Progress in mechanism of ceramics inkjet printing. Journal of Mechanical Engineering, 2017, 53(13): 108-117.

[11] SIVARAMAN K, CHOPRA A, NARAYAN A I, et al. Is zirconia a viable alternative to titanium for oral implant? a critical review. Journal of Prosthodontic Research, 2018, 62(2): 121-133.

[12] DURACCIO D, MUSSANO F, FAGA M G. Biomaterials for dental implants: current and future trends. Journal of Materials Science, 2015, 50(14): 4779-4812.

[13] KOBAYASHI K, KUWAJIMA H, MASAKI T. Phase change and mechanical properties of $\mathrm{ZrO}_{2}-\mathrm{Y}_{2} \mathrm{O}_{3}$ solid electrolyte after ageing. Solid State Ionics, 1981, 3-4: 489-493.

[14] KOSMAČ T, KOCJAN A. Ageing of dental zirconia ceramics. Journal of the European Ceramic Society, 2012, 32(11): 2613-2622.

[15] PEREIRA G K R, VENTURINI A B, SILVESTRI T, et al. Low-temperature degradation of Y-TZP ceramics: a systematic review and meta-analysis. Journal of the Mechanical Behavior of Biomedical Materials, 2016, 55: 151-163.

[16] ÖZCAN M, VOLPATO C Â M, FREDEL M C. Artificial aging of zirconium dioxide: an evaluation of current knowledge and clinical relevance. Current Oral Health Reports, 2016, 3(3): 193-197.

[17] WU Z K, LI N, YAN J Z, et al. Effect of hydrothermal aging on the phase mtability, microstructure and mechanical properties of dental 3Y-TZP ceramics. Applied Mechanics and Materials, 2014, 529: 251-255.

[18] LUGHI V, SERGO V. Low temperature degradation aging of zirconia: a critical review of the relevant aspects in dentistry. Denal
Materials, 2010, 26(8): 807-820.

[19] LANGE F F, DUNLOP G L, DAVIS B I. Degradation during aging of transformationt toughened $\mathrm{ZrO}_{2}-\mathrm{Y}_{2} \mathrm{O}_{3}$ materials at $250{ }^{\circ} \mathrm{C}$. Journal of the American Ceramic Society, 1986, 69(3): 237-240.

[20] SATO T, SHIMADA M. Transformation of yttria-doped tetragonal $\mathrm{ZrO}_{2}$ polycrystals by annealing in water. Journal of the American Ceramic Society, 1985, 68(6): 356-356.

[21] YOSHIMURA M, NOMA T, KAWABATA K, et al. Role of $\mathrm{H}_{2} \mathrm{O}$ on the degradation process of Y-TZP. Journal of Materials Science Letters, 1987, 6(4): 465-467.

[22] GUO X. Hydrothermal degradation mechanism of tetragonal zirconia. Journal of Materials Science, 2001, 36(15): 3737-3744.

[23] GUO X, SCHOBER T. Water incorporation in tetragonal zirconia. Journal of the American Ceramic Society, 2004, 87(4): 746-748.

[24] LANCE M J, VOGEL E M, REITH L A, et al. Low-temperature aging of zirconia ferrules for optical connectors. Journal of the American Ceramic Society, 2001, 84(11): 2731-2733.

[25] HARAGUCHI K, SUGANO N, NISHII T, et al. Phase transformation of a zirconia ceramic head after total hip arthroplasty. The Journal of Bone and Joint Surgery British volume, 2001, 83(7): 996-1000.

[26] CHEVALIER J, GREMILLARD L, VIRKAR A V, et al. The tetragonal-monoclinic transformation in zirconia: Lessons learned and future trends. Journal of the American Ceramic Society, 2009, 92(9): 1901-1920.

[27] CHEVALIER J, CALES B, DROUIN J M. Low-temperature aging of Y-TZP ceramics. Journal of the American Ceramic Society, 2004, 82(8): $2150-2154$.

[28] FABBRI P, PICONI C, BURRESI E, et al. Lifetime estimation of a zirconia-alumina composite for biomedical applications. Dental Materials, 2014, 30(2): 138-142.

[29] CHEVALIER J. What future for zirconia as a biomaterial? Biomaterials, 2006, 27(4): 535-543.

[30] CATTANI-LORENTE M, DURUAL S, AMEZ-DROZ M, et al. Hydrothermal degradation of a 3 Y-TZP translucent dental ceramic: a comparison of numerical predictions with experimental data after 2 years of aging. Dental Materials, 2016, 32(3): 394-402.

[31] WEI C, GREMILLARD L. Towards the prediction of hydrothermal ageing of 3Y-TZP bioceramics from processing parameters. Acta Materialia, 2018, 144: 245-256.

[32] ZHIGACHEV A O, UMRIKHIN A V, RODAEV V V. Theoretical description of zirconia ceramics aging kinetics. Journal of the Australian Ceramic Society, 2018, 55(1): 65-70.

[33] ZHANG F, INOKOSHI M, VANMEENSEL K, et al. Lifetime estimation of zirconia ceramics by linear ageing kinetics. Acta Materialia, 2015, 92: 290-298.

[34] GARVIE R C, NICHOLSON P S. Phase analysis in zirconia systems. Journal of the American Ceramic Society, 1972, 55(6): 303-305.

[35] TORAYA H, YOSHIMURA M, SOMIYA S. Calibration curve for quantitative analysis of the monoclinic-tetragonal $\mathrm{ZrO}_{2}$ system by X-ray diffraction. Journal of the American Ceramic Society, 1984, 67(6): 119-121.

[36] KOYAMA T, KUMAMOTO A, MATSUI K, et al. Revealing tetragonal-to-monoclinic phase transformation in Y-TZP at an initial stage of low temperature degradation using grazing incident-angle X-ray diffraction measurement. Journal of the Ceramic Society of Japan, 2018, 126(9): 728-731.

[37] GREMILLARD L, GRANDJEAN S, CHEVALIER J. A new method to measure monoclinic depth profile in zirconia-based ceramics from X-ray diffraction data. International Journal of Materials Research, 2010, 101(1): 88-94.

[38] CLARKE D R, ADAR F. Measurement of the crystallographically transformed zone produced by fracture in ceramics containing tetragonal zirconia. Journal of the American Ceramic Society, 1982, 65(6): 284-288.

[39] KATAGIRI G, ISHIDA H, ISHITANI A, et al. Direct determination by Raman microprobe of the transformation zone size in $\mathrm{Y}_{2} \mathrm{O}_{3}$ 
containing tetragonal $\mathrm{ZrO}_{2}$ polycrystals. Advances in Ceramics, 1986, 24A: 537-544

[40] LIM C S, FINLAYSON T R, NINIO F, et al. In-situ measurement of the stress-induced phase transformations in magnesia-partially-stabilized zirconia using Raman spectroscopy. Journal of the American Ceramic Society, 1992, 75(6): 1570-1573.

[41] KIM B K, HAHN J W, HAN K R. Quantitative phase analysis in tetragonal-rich tetragonal/monoclinic two phase zirconia by Raman spectroscopy. Journal of Materials Science Letters, 1997, 16(8): 669-671.

[42] CASELlAS D, CUMBRERA F L, SáNCHEZ-BAJO F, et al. On the transformation toughening of $\mathrm{Y}-\mathrm{ZrO}_{2}$ ceramics with mixed Y-TZP/PSZ microstructures. Journal of the European Ceramic Society, 2001, 21(6): 765-777.

[43] LANGE F F. Transformation toughening. Journal of Materials Science, 1982, 17(1): 225-234.

[44] CHEN S Y, LU H Y. Low-temperature ageing map for 3mol\% $\mathrm{Y}_{2} \mathrm{O}_{3}-\mathrm{ZrO}_{2}$. Journal of Materials Science, 1989, 24(2): 453-456.

[45] HALLMANN L, ULMER P, REUSSER E, et al. Effect of dopants and sintering temperature on microstructure and low temperature degradation of dental Y-TZP-zirconia. Journal of the European Ceramic Society, 2012, 32(16): 4091-4104.

[46] PAUL A, VAIDHYANATHAN B, BINNER J G P. Hydrothermal aging behavior of nanocrystalline Y-TZP ceramics. Journal of the American Ceramic Society, 2011, 94(7): 2146-2152.

[47] SWAB J J. Low temperature degradation of Y-TZP materials. Journal of Materials Science, 1991, 26(24): 6706-6714.

[48] LAWSON S, GILL C, DRANSFIELD G P. Hydrothermal and corrosive degradation of Y-TZP ceramics. Key Engineering Materials, 1995, 113: 207-214.

[49] DEVILLE S, CHEVALIER J, FANTOZZI G, et al. Low temperature ageing of zirconia-toughened alumina ceramics and its implication in biomedical implants. Journal of the European Ceramic Society, 2003, 23(15): 2975-2982.

[50] SMIRNOV A, KURLAND H D, GRABOW J, et al. Microstructure, mechanical properties and low temperature degradation resistance of 2Y-TZP ceramic materials derived from nanopowders prepared by laser vaporization. Journal of the European Ceramic Society, 2015, 35(9): 2685-2691.

[51] SUTHARSINI U, THANIHAICHELVAN M, TING $\mathrm{C} \mathrm{H}$, et al. Effect of two-step sintering on the hydrothermal ageing resistance of tetragonal zirconia polycrystals. Ceramics International, 2017, 43(10): 7594-7599.

[52] PRESENDA A, SALVADOR M D, MORENO R, et al. Hydrothermal degradation behavior of Y-TZP ceramics sintered by nonconventional microwave technology. Journal of the American Ceramic Society, 2015, 98(12): 3680-3689.

[53] CHINTAPALLI R, MESTRA A, MARRO F G, et al. stability of nanocrystalline spark plasma sintered 3Y-TZP. Materials, 2010, 3(2): $800-814$

[54] WEI C, GREMILLARD L. Surface treatment methods for mitigation of hydrothermal ageing of zirconia. Journal of the European Ceramic Society, 2019, 39(14): 4322-4329.

[55] DEVILLE S, CHEVALIER J, GREMILLARD L. Influence of surface finish and residual stresses on the ageing sensitivity of biomedical grade zirconia. Biomaterials, 2006, 27(10): 2186-2192.

[56] INOKOSHI M, VANMEENSEL K, ZHANG F, et al. Aging resistance of surface-treated dental zirconia. Dental Materials, 2015, 31(2): 182-194.

[57] WEI C, GREMILLARD L. The influence of stresses on ageing kinetics of 3Y- and 4Y- stabilized zirconia. Journal of the European Ceramic Society, 2018, 38(2): 753-760.

[58] GILES J C. Préparation par reaction à l'état solide et structure des oxynitrures de zirconium. Bulletin de la Société Chimique de France, 1962, 22: 2118-2122.

[59] CHUNG T J, SONG H S, KIM G H, et al. Microstructure and phase stability of yttria-doped tetragonal zirconia polycrystals heat treated in nitrogen atmosphere. Journal of the American Ceramic Society, 1997, 80(10): 2607-2612.

[60] VALLE J, MESTRA A, MARRO F G, et al. Mechanical properties and resistance to low temperature degradation of surface nitrided 3Y-TZP. Journal of the European Ceramic Society, 2013, 33(15/16): 3145-3155.

[61] HÜBSCH C, DELLINGER P, MAIER H J, et al. Protection of yttria-stabilized zirconia for dental applications by oxidic PVD coating. Acta Biomaterialia, 2015, 11(1): 488-493.

[62] SIVAKUMAR S, TEOW H L, SINGH R, et al. The effect of iron oxide on the mechanical and ageing properties of Y-TZP ceramic. Key Engineering Materials, 2016, 701: 225-229.

[63] MAURYA R, GUPTA A, OMAR S, et al. Effect of sintering on mechanical properties of ceria reinforced yttria stabilized zirconia. Ceramics International, 2016, 42(9): 11393-11403.

[64] KHAN M M, RAMESH S, BANG L T, et al. Effect of copper oxide and manganese oxide on properties and low temperature degradation of sintered Y-TZP ceramic. Journal of Materials Engineering and Performance, 2014, 23(12): 4328-4335.

[65] PIVA D H, PIVA R H, ROCHA M C, et al. Resistance of InO1.5-stabilized tetragonal zirconia polycrystals to low-temperature degradation. Materials Letters, 2016, 163: 226-230.

[66] LEE H B, PRINZF B, CAI W. Atomistic simulations of grain boundary segregation in nanocrystalline yttria stabilized zirconia and gadolinia doped ceria solid oxide electrolytes. Acta Materialia, 2013, 61(10): 3872-3887.

[67] YOKOI T, YOSHIYA M, YASUDA H. On modeling of grain boundary segregation in aliovalent cation doped $\mathrm{ZrO}_{2}$ : critical factors in site-selective point defect occupancy. Scripta Materialia, 2015, 102:91-94.

[68] ZHANG F, BATUK M, HADERMANN J, et al. Effect of cation dopant radius on the hydrothermal stability of tetragonal zirconia: grain boundary segregation and oxygen vacancy annihilation. Acta Materialia, 2016, 106: 48-58.

[69] ZHANG F, VANMEENSEL K, INOKOSHI M, et al. Critical influence of alumina content on the low temperature degradation of (2-3)mol\% yttria-stabilized TZP for dental restorations. Journal of the European Ceramic Society, 2015, 35(2): 741-750.

[70] JING Q, BAO J, RUAN F, et al. High-fracture toughness and aging-resistance of $3 \mathrm{Y}-\mathrm{TZP}$ ceramics with a low $\mathrm{Al}_{2} \mathrm{O}_{3}$ content for dental applications. Ceramics International, 2019, 45(5): 6066-6073.

[71] ZHANG F, VANMEENSEL K, BATUK M, et al. Highly-translucent, strong and aging-resistant 3Y-TZP ceramics for dental restoration by grain boundary segregation. Acta Biomaterials, 2015, 16: 215-222.

[72] NAKAMURA T, NAKANO Y, USAMI H, et al. Translucency and low-temperature degradation of silica-doped zirconia: a pilot study. Dental Materials Journal, 2016, 35(4): 571-577.

[73] GREMILLARD L, EPICIER T, CHEVALIER J, et al. Microstructural study of silica-doped zirconia ceramics. Acta Materialia, 2000, 48(18/19): 4647-4652.

[74] SAMODUROVA A, KOCJAN A, SWAIN M V, et al. The combined effect of alumina and silica co-doping on the ageing resistance of 3Y-TZP bioceramics. Acta Biomaterials, 2015, 11: 477-487.

[75] MOHAMED E, TAHERI M, MEHRJOO M, et al. In vitro biocompatibility and ageing of $3 \mathrm{Y}-\mathrm{TZP} / \mathrm{CNTs}$ composites. Ceramics International, 2015, 41(10): 12773-12781.

[76] MORALES-RODRIGUEZ A, POYATO R, GUTIERREZ-MORA $\mathrm{F}$, et al. The role of carbon nanotubes on the stability of tetragonal zirconia polycrystals. Ceramics International, 2018, 44(15): 1771617723.

[77] SONG YAN-JUN, ZHU DONG-BIN, LIANG JIN-SHENG, et al. Enhanced mechanical properties of $3 \mathrm{~mol} \% \quad \mathrm{Y}_{2} \mathrm{O}_{3}$ stabilized tetragonal $\mathrm{ZrO}_{2}$, incorporating tourmaline particles. Ceramics International, 2018, 44(13): 15550-15556.

[78] ZHU DONG-BIN, SONG YAN-JUN, LIANG JIN-SHNEG, et al. Progress of toughness in dental zirconia ceramics. Journal of Inorganic Materials, 2018, 33(4): 363-372. 